\title{
Reduced-order filter for stochastic bilinear systems with multiplicative noise
}

\author{
S. Halabi, H. Souley Ali, H. Rafaralahy, M. Zasadzinski, M. Darouach \\ Université Henri Poincaré - Nancy I, \\ CRAN UMR 7039 - CNRS \\ IUT de Longwy, \\ 186, rue de Lorraine, 54400 Cosnes et Romain, FRANCE \\ e-mail : \{shalabi,mzasad\}@iut-longwy.uhp-nancy.fr
}

\begin{abstract}
This paper deals with the design of a reducedorder $\mathcal{H}_{\infty}$ filter for a stochastic bilinear systems with a prescribed $\mathcal{H}_{\infty}$ norm criterion. The problem is transformed into the search of a unique gain matrix by using a Sylvester-like condition on the drift term. The considered system is bilinear in control and with multiplicative noise in the state and in the measurement equations. The approach is based on the resolution of LMI and is then easily implementable.
\end{abstract}

Keywords - Reduced-order $\mathcal{H}_{\infty}$ filter, Itô's formula, Stochastic systems, Bilinear systems, Lyapunov function.

\section{INTRODUCTION}

The bilinear systems represent sometimes a good mean to physical systems modeling when the linear representation is not sufficiently significant. The stochastic systems get a great importance during the last decades as shown by numerous references (Kozin, 1969; Has'minskii, 1980; Florchinger, 1995; Mao, 1997; Carravetta et al., 2000; Germani et al., 2002; Xu and Chen, 2003).

Generally, bilinear stochastic system designs a stochastic system with multiplicative noise instead of additive one (Carravetta et al., 2000; Germani et al., 2002). The full and the reduced-order $\mathcal{H}_{\infty}$ filtering for stochastic systems with multiplicative noise has been treated in many papers (Hinrichsen and Pritchard, 1998; Gershon et al., 2001; Xu and Chen, 2002; Stoica, 2002). Notice that the measurement equation in (Xu and Chen, 2002; Stoica, 2002) is not corrupted by noise. The problem is solved in terms of two LMIs and a coupling non convex rank constraint.

In this paper the problem of reduced-order $\mathcal{H}_{\infty}$ filtering for a larger class of stochastic systems than those studied in the papers cited above is considered since the studied systems are with multiplicative noise and multiplicative control input (the bilinearity is also between the state and the control input). The measurements are subjected to a multiplicative noise too. Notice that, as in the deterministic case, the multiplicative control input affects the observability of the system.

The purpose is to design a reduced-order $\mathcal{H}_{\infty}$ filter for such a system. We first use a "unbiasedness" (decoupling) condition on the drift part of the estimation error and a change of variable on the control input. Then applying the Itô formula and LMI method permit to reduce the problem to the search of a unique gain matrix. The reduced-order stochastic filter matrices are then computed using this gain.

Throughout the paper, E represents expectation operator with respect to some probability measure $\mathcal{P} .\langle X, Y\rangle=X^{T} Y$ represents the inner product of the vectors $X, Y \in \mathbb{R}^{n}$. herm $(A)$ stands for $A+A^{T}$.

$L_{2}\left(\Omega, \mathbb{R}^{k}\right)$ is the space of square-integrable $\mathbb{R}^{k}$-valued functions on the probability space $(\Omega, \mathcal{F}, \mathcal{P})$ where $\Omega$ is the sample space, $\mathcal{F}$ is a $\sigma$-algebra of subsets of the sample space called events and $\mathcal{P}$ is the probability measure on $\mathcal{F} .\left(\mathcal{F}_{t}\right)_{t \geqslant 0}$ denote an increasing family of $\sigma$-algebras $\left(\mathcal{F}_{t}\right) \in \mathcal{F}$. We also denote by $\widehat{L}_{2}\left([0, \infty) ; \mathbb{R}^{k}\right)$ the space of non-anticipatory square-integrable stochastic process $f()=.(f(t))_{t \in[0, \infty)}$ in $\mathbb{R}^{k}$ with respect to $\left(\mathcal{F}_{t}\right)_{t \in[0, \infty)}$ satisfying

$$
\|f\|_{\widehat{L}_{2}}^{2}=\mathbf{E}\left\{\int_{0}^{\infty}\|f(t)\|^{2} \mathrm{~d} t\right\}<\infty
$$

where $\|$.$\| is the well-known Euclidean norm.$

\section{Problem statement}

Let us consider the following stochastic bilinear system

$$
\left\{\begin{aligned}
\mathrm{d} x(t)= & \left(A_{t 0} x(t)+u_{1}(t) A_{t 1} x(t)\right) \mathrm{d} t \\
& +B_{0} v(t) \mathrm{d} t+A_{w 0} x(t) \mathrm{d} w_{0}(t) \\
\mathrm{d} y(t)= & C x(t) \mathrm{d} t+J_{1} x(t) \mathrm{d} w_{1}(t) \\
z(t)= & L x(t)
\end{aligned}\right.
$$

where $x(t) \in \mathbb{R}^{n}$ is the state vector, $y(t) \in \mathbb{R}^{p}$ is the output, $u_{1}(t) \in \mathbb{R}$ is the control input, $z(t) \in \mathbb{R}^{r}$ is a linear combination of the state vector with $r<n$ and $v(t) \in \mathbb{R}^{q}$ is the perturbation signal. Without loss of generality $L$ is assumed to be a full row rank matrix. $w_{i}(t)$ is a Wiener process verifying (Has'minskii, 1980)

$$
\begin{gathered}
\mathbf{E}\left(\mathrm{d} w_{i}(t)\right)=0, \mathbf{E}\left(\mathrm{d} w_{i}(t)^{2}\right)=\mathrm{d} t, \text { for } i=0,1, \\
\mathbf{E}\left(\mathrm{d} w_{0}(t) \mathrm{d} w_{1}(t)\right)=\mathbf{E}\left(\mathrm{d} w_{1}(t) \mathrm{d} w_{0}(t)\right)=\varphi \mathrm{d} t, \\
\text { with }|\varphi|<1 .
\end{gathered}
$$

As in the most cases for physical processes, we assume that the stochastic bilinear system (1) has known bounded control input, i.e. $u_{1}(t) \in \Gamma \subset \mathbb{R}$, where

$$
\Gamma=\left\{u_{1}(t) \in \mathbb{R} \mid u_{1 \min } \leqslant u_{1}(t) \leqslant u_{1 \max }\right\} .
$$

The study made here can be easily generalized for the case where there are $m$ control inputs.

First, we introduce the following definition and assumption.

Definition 1. (Kozin, 1969; Has'minskii, 1980) The stochastic system (1) with $v(t) \equiv 0$ is said to be asymptotically mean-square stable if all initial states $x(0)$ yields

$$
\lim _{t \rightarrow \infty} \mathbf{E}\|x(t)\|^{2}=0, \quad \forall u_{1}(t) \in \Gamma .
$$


Assumption 1. The stochastic bilinear system (1) is assumed to be asymptotically mean-square stable.

In this paper, the aim is to design a reduced-order filter in the following form

$$
\begin{aligned}
& \mathrm{d} \eta(t)=\left(M_{0}+u_{1}(t) M_{1}\right) \eta(t) \mathrm{d} t \\
&+\left(N_{0}+u_{1}(t) N_{1}\right) \mathrm{d} y(t)
\end{aligned}
$$

where $\eta(t) \in \mathbb{R}^{r}$ is the filter state with $r<n$ and the matrices $M_{i}$ and $N_{i}$ (for $i=0,1$ ) are to be determined.

Then the following problem is considered.

Problem 1. Given a real $\gamma>0$, the goal is to design a asymptotically mean-square stable reduced-order $\mathcal{H}_{\infty}$ filter (5) such that the augmented state $\left[x^{T}(t) e^{T}(t)\right]^{T}$ is asymptotically mean-square stable and the following $\mathcal{H}_{\infty}$ performance

$$
\|e(t)\|_{\widehat{L}_{2}}^{2} \leqslant \gamma\|v(t)\|_{\widehat{L}_{2}}^{2}
$$

is achieved from the disturbance $v(t)$ to the filtering error $e(t)=z(t)-\eta(t)$

Let us consider the following estimation error

$$
e(t)=L x(t)-\eta(t)
$$

So the estimation error dynamics becomes

$$
\begin{aligned}
\mathrm{d} e(t)= & \left(M_{0}+M_{1} u_{1}(t)\right) e(t) \mathrm{d} t+L B_{0} v(t) \mathrm{d} t \\
+ & \left\{\left(L A_{t 0}-M_{0} L-N_{0} C\right)\right. \\
& \left.+\left(L A_{t 1}-M_{1} L-N_{1} C\right) u_{1}(t)\right\} x(t) \mathrm{d} t \\
& +L A_{w 0} x(t) \mathrm{d} w_{0}(t) \\
& -\left(\left(N_{0}+u_{1}(t) N_{1}\right) J_{1} x(t) \mathrm{d} w_{1}(t) .\right.
\end{aligned}
$$

In order to supress the direct effect of the state $x(t)$ on the drift part of the filtering error, we consider the following Sylvester-like conditions

$$
L A_{t i}-M_{i} L-N_{i} C=0, \quad i=0,1 .
$$

Let us consider the following augmented state vector

$$
\xi^{T}(t)=\left[x^{T}(t) e^{T}(t)\right] .
$$

Then under (9), the dynamics of the augmented system is given by

$$
\begin{aligned}
\mathrm{d} \xi(t)= & \left(\mathcal{A}_{t 0}+\mathcal{A}_{t 1} u_{1}(t)\right) \xi(t) \mathrm{d} t+\mathcal{B}_{0} v(t) \mathrm{d} t \\
+ & \mathcal{A}_{w 0} \xi(t) \mathrm{d} w_{0}(t) \\
& +\left(\mathcal{A}_{w 1}+\mathcal{A}_{w 2} u_{1}(t)\right) \xi(t) \mathrm{d} w_{1},
\end{aligned}
$$

with

$$
\begin{aligned}
\mathcal{A}_{t i} & =\left[\begin{array}{cc}
A_{t i} & 0 \\
0 & M_{i}
\end{array}\right], & \text { for } & i=0,1, \\
\mathcal{B}_{0} & =\left[\begin{array}{c}
B_{0} \\
L B_{0}
\end{array}\right], & \mathcal{A}_{w 0} & =\left[\begin{array}{cc}
A_{w 0} & 0 \\
L A_{w 0} & 0
\end{array}\right], \\
\mathcal{A}_{w 1} & =\left[\begin{array}{cc}
0 & 0 \\
-N_{0} J_{1} & 0
\end{array}\right], & \mathcal{A}_{w 2} & =\left[\begin{array}{cc}
0 & 0 \\
-N_{1} J_{1} & 0
\end{array}\right] .
\end{aligned}
$$

In the sequel the relations (9) are used to express the filter matrices through a single gain matrix.
In fact, since $L$ is a full row rank matrix, relations (9) are equivalent to

$$
\begin{array}{r}
\left(L A_{t i}-M_{i} L-N_{i} C\right)\left[L^{\dagger} \quad\left(I_{n}-L^{\dagger} L\right)\right]=0, \\
\text { for } \quad i=0,1 .
\end{array}
$$

where $L^{\dagger}$ is a generalized inverse of matrix $L$ satisfying $L=$ $L L^{\dagger} L$ (Lancaster and Tismenetsky, 1985) (since $\operatorname{rank} L=r$, we have $\left.L L^{\dagger}=I_{r}\right)$.

Relations (13) give

$$
\begin{aligned}
& 0=L A_{t i} L^{\dagger}-M_{i}-N_{i} C L^{\dagger} \quad \text { for } i=0,1, \\
& 0=L \bar{A}_{i}-N_{i} \bar{C} \quad \text { for } \quad i=0,1,
\end{aligned}
$$

where

$$
\begin{aligned}
\bar{A}_{i} & =A_{t i}\left(I_{n}-L^{\dagger} L\right) \quad \text { for } \quad i=0,1, \\
\bar{C} & =C\left(I_{n}-L^{\dagger} L\right) .
\end{aligned}
$$

The relation $(14 \mathrm{a})$ gives

$$
M_{i}=\overline{\bar{A}}_{i}-N_{i} \overline{\bar{C}}, \quad \text { for } \quad i=0,1,
$$

where

$$
\begin{aligned}
\overline{\bar{A}}_{i} & =L A_{t i} L^{\dagger}, \quad \text { for } \quad i=0,1, \\
\overline{\bar{C}} & =C L^{\dagger} .
\end{aligned}
$$

The relation $(14 \mathrm{~b})$ becomes

$$
\mathcal{K} \Sigma=L \bar{A}
$$

where

$$
\begin{aligned}
& \mathcal{K}=\left[\begin{array}{ll}
N_{0} & N_{1}
\end{array}\right], \\
& \bar{A}=\left[\begin{array}{ll}
\bar{A}_{0} & \bar{A}_{1}
\end{array}\right], \\
& \Sigma=\left[\begin{array}{ll}
\bar{C} & 0 \\
0 & \bar{C}
\end{array}\right],
\end{aligned}
$$

and a general solution to equation (18), if it exists, is given by

$$
\mathcal{K}=L \bar{A} \Sigma^{\dagger}+Z\left(I_{2 p}-\Sigma \Sigma^{\dagger}\right),
$$

where

$$
Z=\left[\begin{array}{ll}
Z_{0} & Z_{1}
\end{array}\right]
$$

is an arbitrary matrix of appropriate dimensions.

\section{TRANFORMATION OF THE BILINEAR SYSTEM FILTERING PROBLEM INTO AN UNCERTAIN ONE}

As in (Zasadzinski et al., 2003), let us introduce a change of variable on the control $u_{1}(t)$ as follows

$$
u_{1}(t)=\alpha_{1}+\sigma_{1} \varepsilon_{1}(t)
$$

where $\alpha_{1} \in \mathbb{R}$ and $\sigma_{1} \in \mathbb{R}$ are given by

$$
\alpha_{1}=\frac{1}{2}\left(u_{1 \min }+u_{1 \max }\right), \sigma_{1}=\frac{1}{2}\left(u_{1 \max }-u_{1 \min }\right) .
$$

The new "uncertain" variable is $\varepsilon_{1}(t) \in \bar{\Gamma} \subset \mathbb{R}$ where the polytope $\bar{\Gamma}$ is defined by

$$
\bar{\Gamma}=\left\{\varepsilon_{1}(t) \in \mathbb{R} \mid \varepsilon_{1 \min }=-1 \leqslant \varepsilon_{1}(t) \leqslant \varepsilon_{1 \max }=1\right\} .
$$


Then the error dynamics (8) can be rewritten as

$$
\begin{aligned}
& \mathrm{d} e(t)=\left(\mathbb{A}_{t}-Z \mathbb{C}_{t}+\left(\widetilde{\mathbb{A}}_{t}-Z \widetilde{\mathbb{C}}_{t}\right) \Delta_{\varepsilon}\left(\varepsilon_{1}(t)\right) H_{e}\right) e(t) \mathrm{d} t \\
& +\mathbb{B}_{0} v(t) \mathrm{d} t+\mathbb{A}_{w 0} x(t) \mathrm{d} w_{0}(t)+\left(\mathbb{A}_{w(11)}-Z \mathbb{A}_{w(12)}\right. \\
& \left.+\left(\widetilde{\mathbb{A}}_{w(11)}-Z \widetilde{\mathbb{A}}_{w(12)}\right) \Delta_{x}\left(\varepsilon_{1}(t)\right) H_{x}\right) x(t) \mathrm{d} w_{1}(t)
\end{aligned}
$$

where

$$
\begin{array}{lc}
\mathbb{A}_{t}=\overline{\bar{A}}_{0}+\alpha_{1} \overline{\bar{A}}_{1}-L \bar{A} \Sigma^{\dagger} \Lambda, & \mathbb{C}_{t}=\left(I_{2 p}-\Sigma \Sigma^{\dagger}\right) \Lambda, \\
\widetilde{\mathbb{A}}_{t}=\sigma_{1} \overline{\bar{A}}_{1}-L \bar{A} \Sigma^{\dagger} \Lambda, & \widetilde{\mathbb{C}}_{t}=\left(I_{2 p}-\Sigma \Sigma^{\dagger}\right) \Lambda, \\
\mathbb{B}_{01}=L B_{0}, & \mathbb{A}_{w 0}=L A_{w 0}, \\
\mathbb{A}_{w(11)}=L \bar{A} \Sigma^{\dagger} \Psi_{\alpha}, & \mathbb{A}_{w(12)}=\left(I_{2 p}-\Sigma \Sigma^{\dagger}\right) \Psi_{\alpha}, \\
\widetilde{\mathbb{A}}_{w(11)}=L \bar{A} \Sigma^{\dagger} \Psi_{\sigma}, & \widetilde{\mathbb{A}}_{w(12)}=\left(I_{2 p}-\Sigma \Sigma^{\dagger}\right) \Psi_{\sigma},
\end{array}
$$

and

$$
\begin{gathered}
\Lambda=\left[\begin{array}{c}
C L^{\dagger} \\
\alpha_{1} C L^{\dagger}
\end{array}\right], \quad \Psi_{\alpha}=\left[\begin{array}{c}
-J_{1} \\
-\alpha J_{1}
\end{array}\right], \quad \Psi_{\sigma}=\left[\begin{array}{c}
0 \\
-\sigma J_{1}
\end{array}\right], \\
H_{e}=I_{r}, \quad I_{n}, \\
\Delta_{\varepsilon}\left(\varepsilon_{1}(t)\right)=\varepsilon_{1}(t) I_{r}, \quad \Delta_{x}\left(\varepsilon_{1}(t)\right)=\varepsilon_{1}(t) I_{n} .
\end{gathered}
$$

Using the definition (26), the matrix $\Delta_{\varepsilon}\left(\varepsilon_{1}(t)\right)$ and $\Delta_{x}\left(\varepsilon_{1}(t)\right)$ satisfy

$$
\left\|\Delta_{\varepsilon_{1}}\left(\varepsilon_{1}(t)\right)\right\| \leqslant 1, \quad \text { and } \quad\left\|\Delta_{x}\left(\varepsilon_{1}(t)\right)\right\| \leqslant 1 .
$$

Using (24), the system state equation (see (1)) becomes

$$
\begin{aligned}
\mathrm{d} x(t)=\left(A_{t 0}+\alpha_{1} A_{t 1}\right. & \left.+\sigma_{1} \varepsilon_{1}(t) A_{t 1}\right) x(t) \mathrm{d} t \\
& +B_{0} v(t) \mathrm{d} t+A_{w 0} x(t) \mathrm{d} w_{0}(t) .
\end{aligned}
$$

So the augmented system (11) is rewritten as

$$
\begin{aligned}
\mathrm{d} \xi(t)= & \left(\widehat{A}_{t 0}+\Delta \widehat{A}_{t 0}(t)\right) \xi(t) \mathrm{d} t+\widehat{B}_{0} v(t) \mathrm{d} t \\
& +\widehat{A}_{w 0} \xi(t) \mathrm{d} w_{0}(t) \\
& +\left(\widehat{A}_{w 1}+\Delta \widehat{A}_{w 1}(t)\right) \xi(t) \mathrm{d} w_{1}(t)
\end{aligned}
$$

where

$$
\begin{aligned}
& \widehat{A}_{t 0}=\left[\begin{array}{cc}
A_{t 0}+\alpha_{1} A_{t 1} & 0 \\
0 & \mathbb{A}_{t}-Z \mathbb{C}_{t}
\end{array}\right], \\
& \Delta \widehat{A}_{t 0}(t)=H_{1} \Delta_{\xi}\left(\varepsilon_{1}(t)\right) H_{t}, \\
& \widehat{B}_{0}=\left[\begin{array}{c}
B_{0} \\
\mathbb{B}_{01}
\end{array}\right], \quad \widehat{A}_{w 0}=\left[\begin{array}{ll}
A_{w 0} & 0 \\
\mathbb{A}_{w 0} & 0
\end{array}\right], \\
& \widehat{A}_{w 1}=\left[\begin{array}{cc}
0 & 0 \\
\mathbb{A}_{w(11)}-Z \mathbb{A}_{w(12)} & 0
\end{array}\right], \\
& \Delta \widehat{A}_{w 1}(t)=H_{2} \Delta_{\xi}\left(\varepsilon_{1}(t)\right) H_{w}, \\
& H_{1}=\left[\begin{array}{cc}
\sigma_{1} A_{t 1} & 0 \\
0 & \widetilde{\mathbb{A}}_{t}-Z \widetilde{\mathbb{C}}_{t}
\end{array}\right], H_{2}=\left[\begin{array}{c}
0 \\
\widetilde{\mathbb{A}}_{w(11)}-Z \widetilde{\mathbb{A}}_{w(12)}
\end{array}\right], \\
& H_{t}=\left[\begin{array}{cc}
I_{n} & 0 \\
0 & I_{r}
\end{array}\right], \quad H_{w}=\left[\begin{array}{ll}
I_{n} & 0
\end{array}\right], \Delta_{\xi}\left(\varepsilon_{1}(t)\right)=\varepsilon_{1}(t) .
\end{aligned}
$$

Notice that from $(26), \Delta_{\xi}\left(\varepsilon_{1}(t)\right)$ satisfy

$$
\left\|\Delta_{\xi}\left(\varepsilon_{1}(t)\right)\right\| \leqslant 1
$$

\section{Synthesis of the REDUCED-ORDER FILTER}

Consider the following system obtained from (30)

$$
\left\{\begin{array}{l}
\mathrm{d} \xi(t)=\left(\widehat{A}_{t 0}+\Delta \widehat{A}_{t 0}(t)\right) \xi(t) \mathrm{d} t+\widehat{B}_{0} v(t) \mathrm{d} t \\
+\widehat{A}_{w 0} \xi(t) \mathrm{d} w_{0}(t)+\left(\widehat{A}_{w 1}+\Delta \widehat{A}_{w 1}(t)\right) \xi(t) \mathrm{d} w_{1}(t) \\
e(t)=\widehat{C} \xi(t)
\end{array}\right.
$$

where $\widehat{C}=\left[\begin{array}{ll}0 & I_{r}\end{array}\right]$.

Then the following theorem is given for the filter synthesis.

Theorem 1. The reduced-order $\mathcal{H}_{\infty}$ filtering problem 1 is solved for the system (1) with the filter (5) such that the augmented system (32) is asymptotically mean-square stable and verifies the $\mathcal{H}_{\infty}$ performance (6) if, for some reals $\mu_{1}>0, \mu_{2}>0$ and $\mu_{3}>0$ there exist matrices $P_{1}=P_{1}^{T}>$ $0 \in \mathbb{R}^{n \times n}, P_{2}=P_{2}^{T}>0 \in \mathbb{R}^{r \times r}, P_{3} \in \mathbb{R}^{n \times r}, G_{2} \in \mathbb{R}^{r \times 2 p}$ and $G_{3} \in \mathbb{R}^{n \times 2 p}$ such that

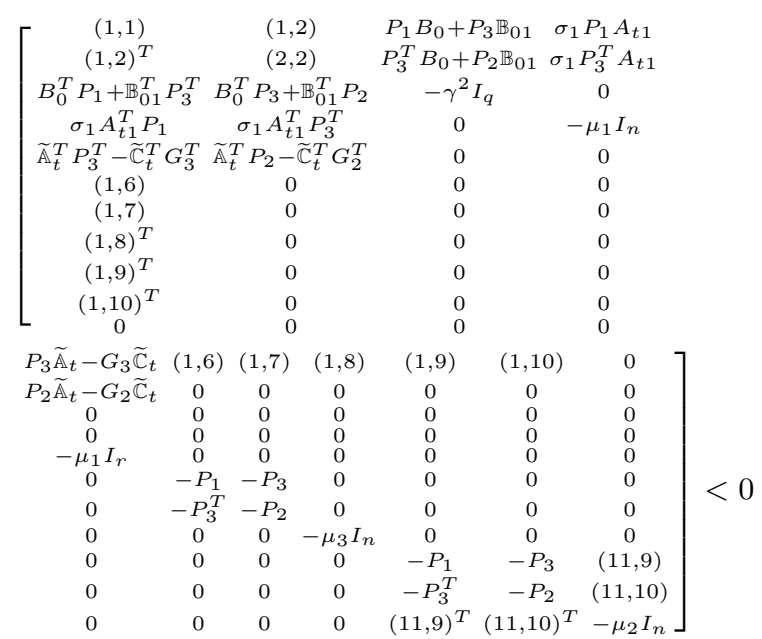

where

$$
\begin{aligned}
& (1,1)=\left(\mu_{1}+\mu_{2}+\varphi \mu_{3}\right) I_{n}+\operatorname{herm}\left\{P_{1} A_{\alpha 1}\right. \\
& +\varphi\left(A_{w 0}^{T}\left(P_{3} \mathbb{A}_{w(11)}-G_{3} \mathbb{A}_{w(12)}\right)\right. \\
& \left.\left.+\mathbb{A}_{w 0}^{T}\left(P_{2} \mathbb{A}_{w(11)}-G_{2} \mathbb{A}_{w(12)}\right)\right)\right\}, \\
& (1,2)=A_{\alpha 1}^{T} P_{3}+P_{3} \mathbb{A}_{t}-G_{3} \mathbb{C}_{t}, \\
& (2,2)=\operatorname{herm}\left(P_{2} \mathbb{A}_{t}-G_{2} \mathbb{C}_{t}\right)+\left(1+\mu_{1}\right) I_{r}, \\
& (1,6)=A_{w 0}^{T} P_{1}+\mathbb{A}_{w 0}^{T} P_{3}^{T}, \\
& (1,7)=A_{w 0}^{T} P_{3}+\mathbb{A}_{w 0}^{T} P_{2}, \\
& (1,8)=\varphi^{\frac{1}{2}}\left(A_{w 0}^{T}\left(P_{3} \widetilde{\mathbb{A}}_{w(11)}-G_{3} \widetilde{\mathbb{A}}_{w(12)}\right)\right. \\
& \left.+\mathbb{A}_{w 0}^{T}\left(P_{2} \widetilde{\mathbb{A}}_{w(11)}-G_{2} \widetilde{\mathbb{A}}_{w(12)}\right)\right), \\
& (1,9)=\mathbb{A}_{w(11)}^{T} P_{3}^{T}-\mathbb{A}_{w(12)}^{T} G_{3}^{T}, \\
& (1,10)=\mathbb{A}_{w(11)}^{T} P_{2}-\mathbb{A}_{w(12)}^{T} G_{2}^{T}, \\
& (11,9)=P_{3} \widetilde{\mathbb{A}}_{w(11)}-G_{3} \widetilde{\mathbb{A}}_{w(12)}, \\
& (11,10)=P_{2} \widetilde{\mathbb{A}}_{w(11)}-G_{2} \widetilde{\mathbb{A}}_{w(12)}, \\
& A_{\alpha 1}=A_{t 0}+\alpha_{1} A_{t 1},
\end{aligned}
$$

and such that the gain matrices $G_{2}$ and $G_{3}$ are the solution of the following equation

$$
\left[\begin{array}{l}
G_{2} \\
G_{3}
\end{array}\right]=\left[\begin{array}{l}
P_{2} \\
P_{3}
\end{array}\right] Z .
$$


Proof. Consider the following Lyapunov function

$$
V(\xi)=\xi^{T} \mathcal{P} \xi
$$

where

$$
\mathcal{P}=\left[\begin{array}{ll}
P_{1} & P_{3} \\
P_{3}^{T} & P_{2}
\end{array}\right] .
$$

Applying Itô formula (Mao, 1997) to the system (30) (or (32)), we get

$$
\mathrm{d} V(\xi(t))=\mathcal{L} V(\xi(t)) \mathrm{d} t+2 \xi^{T}(t) \mathcal{P} \Psi(t) \xi(t)
$$

where

$\Psi(t)=\widehat{A}_{w 0} \mathrm{~d} w_{0}(t)+\left(\widehat{A}_{w 1}+\Delta \widehat{A}_{w 1}(t)\right) \mathrm{d} w_{1}(t)$,

and

$$
\begin{array}{r}
\mathcal{L} V(\xi(t)) \mathrm{d} t=2 \xi^{T}(t)\left(\left(\widehat{A}_{t 0}+\Delta \widehat{A}_{t 0}(t)\right) \xi(t)+\widehat{B}_{0} v(t)\right) \mathrm{d} t \\
+\xi^{T}(t)\langle\mathcal{P} \Psi(t), \Psi(t)\rangle \xi(t) .
\end{array}
$$

By replacing (38) and (39), the relation (37) becomes

$$
\begin{aligned}
& \mathrm{d} V(\xi(t))= \\
& 2 \xi^{T}(t) \mathcal{P}\left(\left(\widehat{A}_{t 0}+\Delta \widehat{A}_{t 0}(t)\right) \xi(t)+\widehat{B}_{0} v(t)\right) \mathrm{d} t \\
& +\xi^{T}(t) \widehat{A}_{w 0}^{T} \mathcal{P} \widehat{A}_{w 0} \xi(t) \mathrm{d} w_{0}(t)^{2} \\
& +\xi^{T}(t)\left(\widehat{A}_{w 1}+\Delta \widehat{A}_{w 1}(t)\right)^{T} \mathcal{P} \\
& \times\left(\widehat{A}_{w 1}+\Delta \widehat{A}_{w 1}(t)\right) \xi(t) \mathrm{d} w_{1}(t)^{2} \\
& +\xi^{T}(t) \widehat{A}_{w 0}^{T} \mathcal{P}\left(\widehat{A}_{w 1}+\Delta \widehat{A}_{w 1}(t)\right) \xi(t) \mathrm{d} w_{0}(t) \mathrm{d} w_{1}(t) \\
& +\xi^{T}(t)\left(\widehat{A}_{w 1}+\Delta \widehat{A}_{w 1}(t)\right)^{T} \mathcal{P} \widehat{A}_{w 0} \xi(t) \mathrm{d} w_{1}(t) \mathrm{d} w_{0}(t) \\
& +2 \xi^{T}(t) \mathcal{P} \widehat{A}_{w 0} \xi(t) \mathrm{d} w_{0}(t) \\
& +2 \xi^{T}(t) \mathcal{P}\left(\widehat{A}_{w 1}+\Delta \widehat{A}_{w 1}(t)\right) \xi(t) \mathrm{d} w_{1}(t) .
\end{aligned}
$$

Using the majoration lemma (Wang et al., 1992), it can be shown that

$$
\begin{aligned}
& 2 \xi^{T}(t) \mathcal{P} \Delta \widehat{A}_{t 0}(t) \xi(t) \\
& \quad \leqslant \xi^{T}(t)\left(\mu_{1}^{-1} \mathcal{P} H_{1} H_{1}^{T} \mathcal{P}+\mu_{1} H_{t}^{T} H_{t}\right) \xi(t) \\
& \left(\widehat{A}_{w 1}+\Delta \widehat{A}_{w 1}(t)\right)^{T} \mathcal{P}\left(\widehat{A}_{w 1}+\Delta \widehat{A}_{w 1}(t)\right) \\
& \quad \leqslant \widehat{A}_{w 1}^{T}\left(\mathcal{P}^{-1}-\mu_{2}^{-1} H_{2} H_{2}^{T}\right)^{-1} \widehat{A}_{w 1}+\mu_{2} H_{w}^{T} H_{w} \\
& 2 \xi^{T}(t) \widehat{A}_{w 0}^{T} \mathcal{P} \Delta \widehat{A}_{w 1}(t) \xi(t) \\
& \leqslant \xi^{T}(t)\left(\mu_{3}^{-1} \widehat{A}_{w 0}^{T} \mathcal{P} H_{2} H_{2}^{T} \mathcal{P} \widehat{A}_{w 0}+\mu_{3} H_{w}^{T} H_{w}\right) \xi(t) .
\end{aligned}
$$

Now, taking the expectation of (40) (see (Mao, 1997)) and using the relations (2) and the last three inequalities, then $\mathbf{E}\{\mathrm{d} V(\xi(t))\}$ can be bounded as

$$
\mathbf{E}\{\mathrm{d} V(\xi(t))\} \leqslant \mathbf{E}\left\{\left[\xi(t)^{T} v(t)^{T}\right] \Theta_{1}\left[\begin{array}{l}
\xi(t) \\
v(t)
\end{array}\right] \mathrm{d} t\right\}
$$

where

$$
\begin{aligned}
& \Theta_{1}=\left[\begin{array}{cc}
\mathcal{P} \widehat{A}_{t 0}+\widehat{A}_{t 0}^{T} \mathcal{P}+\mathcal{C}^{T} \mathcal{C} & \mathcal{P} \widehat{B}_{0} \\
\widehat{B}_{0}^{T} \mathcal{P} & -\gamma^{2} I_{q}
\end{array}\right] \\
& +\mu_{1}\left[\begin{array}{cc}
H_{t}^{T} H_{t} & 0 \\
0 & 0
\end{array}\right]+\mu_{1}^{-1}\left[\begin{array}{cc}
\mathcal{P} H_{1}^{T} H_{1} \mathcal{P} & 0 \\
0 & 0
\end{array}\right] \\
& +\left[\begin{array}{cc}
\widehat{A}_{w 1}^{T}\left(\mathcal{P}^{-1}-\mu_{2}^{-1} H_{2} H_{2}^{T}\right)^{-1} \widehat{A}_{w 1} & 0 \\
0 & 0
\end{array}\right]+\mu_{2}\left[\begin{array}{cc}
H_{w}^{T} H_{w} & 0 \\
0 & 0
\end{array}\right] \\
& +\varphi \mu_{3}\left[\begin{array}{cc}
H_{w}^{T} H_{w} & 0 \\
0 & 0
\end{array}\right]+\left[\begin{array}{cc}
\widehat{A}_{w 0}^{T} \mathcal{P} \widehat{A}_{w 0} & 0 \\
0 & 0
\end{array}\right] \\
& +\left[\begin{array}{cc}
\varphi \mu_{3}^{-1}\left(\widehat{A}_{w 0}^{T} \mathcal{P} H_{2} H_{2}^{T} \mathcal{P} \widehat{A}_{w 0}\right) & 0 \\
0 & 0
\end{array}\right] \\
& +\varphi\left[\begin{array}{cc}
\widehat{A}_{w 0}^{T} \mathcal{P} \widehat{A}_{w 1}+\widehat{A}_{w 1}^{T} \mathcal{P} \widehat{A}_{w 0} & 0 \\
0 & 0
\end{array}\right] .
\end{aligned}
$$

Now, applying the Schur lemma (Boyd et al., 1994), $\Theta_{1}$ can be rewritten as

$$
\begin{aligned}
& {\left[\begin{array}{cccc}
(1,1) & \mathcal{P} \widehat{B}_{0} & \mathcal{P} H_{1} & \widehat{A}_{w 0}^{T} \mathcal{P} \\
\widehat{B}_{0}^{T} \mathcal{P} & -\gamma^{2} I_{q} & 0 & 0 \\
H_{1}^{T} \mathcal{P} & 0 & -\mu_{1} I_{n+r} & 0 \\
\mathcal{P} \widehat{A}_{w 0} & 0 & 0 & -\mathcal{P} \\
\varphi^{\frac{1}{2}} H_{2}^{T} \mathcal{P} \widehat{A}_{w 0} & 0 & 0 & 0 \\
\mathcal{P} \widehat{A}_{w 1} & 0 & 0 & 0 \\
0 & 0 & 0 & 0
\end{array}\right.} \\
& \left.\begin{array}{ccc}
\varphi^{\frac{1}{2}} \widehat{A}_{w 0}^{T} \mathcal{P} H_{2} & \widehat{A}_{w 1}^{T} \mathcal{P} & 0 \\
0 & 0 & 0 \\
0 & 0 & 0 \\
0 & 0 & 0 \\
-\mu_{3} I_{n+r} & 0 & 0 \\
0 & -\mathcal{P} & \mathcal{P} H_{2} \\
0 & H_{2}^{T} \mathcal{P} & -\mu_{2} I_{n}
\end{array}\right]
\end{aligned}
$$

where

$$
\begin{aligned}
(1,1)=\mathcal{P} \widehat{A}_{t 0}+\widehat{A}_{t 0}^{T} \mathcal{P}+\widehat{C}^{T} \widehat{C}+\left(\mu_{2}+\varphi \mu_{3}\right) H_{w}^{T} H_{w} \\
+\mu_{1} H_{t}^{T} H_{t}+\varphi\left(\widehat{A}_{w 0}^{T} \mathcal{P} \widehat{A}_{w 1}+\widehat{A}_{w 1}^{T} \mathcal{P} \widehat{A}_{w 0}\right) .
\end{aligned}
$$

Once the LMI (33) is verified, the asymptotic mean-square stability of the system $(32)$, for $v(t) \equiv 0$, can be proved using Schur lemma and the same method of (Souley Ali et al., 2005).

Now consider the following performance index

$J_{\xi v}=\int_{0}^{\infty} \mathbf{E}\left(\xi^{T}(t) \widehat{C}^{T} \widehat{C} \xi(t)-\gamma^{2} v^{T}(t) v(t)\right) \mathrm{d} t$.

Writting $J_{\xi v}$ as

$$
\begin{aligned}
J_{\xi v}=\int_{0}^{\infty} & \left\{\mathbf { E } \left(\left(\xi^{T}(t) \widehat{C}^{T} \widehat{C} \xi(t)-\gamma^{2} v^{T}(t) v(t)\right) \mathrm{d} t\right.\right. \\
+ & \mathrm{d} V(\xi(t)))\}-\mathbf{E}\left(V(\xi(t))_{t=\infty}+\mathbf{E}\left(V(\xi(t))_{t=0} .\right.\right.
\end{aligned}
$$

Or, since $\mathbf{E}\left(V(\xi(t))_{t=0}=0\right.$ because $\xi(0)=0$ and $\mathbf{E}\left(V(\xi(t))_{t=\infty} \geqslant 0\right.$, this implies

$$
\begin{array}{r}
J_{\xi v} \leqslant \int_{0}^{\infty}\left\{\mathbf { E } \left(\left(\xi^{T}(t) \widehat{C}^{T} \widehat{C} \xi(t)-\gamma^{2} v^{T}(t) v(t)\right) \mathrm{d} t\right.\right. \\
+\mathrm{d} V(\xi(t)))\} .
\end{array}
$$


Now if the LMI (33) holds, then applying Schur lemma yields

$$
\underbrace{\left[\begin{array}{cc}
\Theta & \mathcal{P} \widehat{B}_{0} \\
\widehat{B}_{0}^{T} \mathcal{P} & 0
\end{array}\right]}_{\Pi}+\left[\begin{array}{cc}
\widehat{C}^{T} \widehat{C} & 0 \\
0 & -\gamma^{2} I_{q}
\end{array}\right]<0
$$

with

$$
\begin{aligned}
\Theta=\mathcal{P} \widehat{A}_{t 0}+\widehat{A}_{t 0}^{T} \mathcal{P}+\mu_{1} H_{t}^{T} H_{t}+\left(\mu_{2}+\varphi \mu_{3}\right) H_{w}^{T} H_{w} \\
+\varphi\left(\widehat{A}_{w 0}^{T} \mathcal{P} \widehat{A}_{w 1}+\widehat{A}_{w 1}^{T} \mathcal{P} \widehat{A}_{w 0}\right)+\mu_{1}^{-1} \mathcal{P} H_{1}^{T} H_{1} \mathcal{P}+ \\
\widehat{A}_{w 0}^{T} \mathcal{P} \widehat{A}_{w 0}+\varphi \mu_{3}^{-1}\left(\widehat{A}_{w 0}^{T} \mathcal{P} H_{2} H_{2}^{T} \mathcal{P} \widehat{A}_{w 0}\right)
\end{aligned}
$$

Therefore

$$
\begin{aligned}
J_{\xi v} \leqslant \int_{0}^{\infty} & \mathbf{E}\left(\left[\begin{array}{ll}
\xi(t)^{T} & v(t)^{T}
\end{array}\right] \Pi\left[\begin{array}{l}
\xi(t) \\
v(t)
\end{array}\right] \mathrm{d} t\right. \\
& \left.+\left[\begin{array}{ll}
\xi(t)^{T} & v(t)^{T}
\end{array}\right]\left[\begin{array}{cc}
\widehat{C}^{T} \widehat{C} & 0 \\
0 & -\gamma^{2} I_{q}
\end{array}\right]\left[\begin{array}{l}
\xi(t) \\
v(t)
\end{array}\right] \mathrm{d} t\right)<0,
\end{aligned}
$$

so, if the LMI (33) holds the asymptotic mean-square stability and the $\mathcal{H}_{\infty}$ performance are proved.

\section{Numerical EXAMPLE}

Consider the stochastic bilinear system (1) and suppose that the matrices have the following numerical value

$$
\begin{aligned}
& A_{t 0}=\left[\begin{array}{ccc}
-1.5 & 1 & -1 \\
0.5 & -2.5 & 1 \\
0 & -0.6 & -3.5
\end{array}\right], \quad B_{0}=\left[\begin{array}{cc}
-0.1 & 0.3 \\
-1 & 0.2 \\
0.6 & 0.5
\end{array}\right] \text {, } \\
& A_{t 1}=\left[\begin{array}{ccc}
-0.01 & 0.1 & 0 \\
0 & -0.05 & 0 \\
0.15 & 0 & -0.02
\end{array}\right] \text {, } \\
& A_{w 0}=\left[\begin{array}{ccc}
1 & 0 & 0.2 \\
0.5 & 0.3 & -0.1 \\
-0.2 & 0 & 0.2
\end{array}\right] \text {, } \\
& C=\left[\begin{array}{lll}
1 & 0 & 0 \\
0 & 1 & 0
\end{array}\right], \quad L=\left[\begin{array}{ccc}
0 & -1 & 1 \\
1 & 0 & -1
\end{array}\right], \\
& J_{1}=\left[\begin{array}{ccc}
-0.03 & 0 & -0.03 \\
0 & -0.01 & 0
\end{array}\right] \text {. }
\end{aligned}
$$

The control $u_{1}(t)$ is defined as in (3), with

$$
u_{1 \min }=-5 \leqslant u_{1}(t) \leqslant u_{1 \max }=6,
$$

and the initial state $\xi(0)=\left[\begin{array}{ll}x^{T}(0) & e^{T}(0)\end{array}\right]^{T}$ is

$$
\xi(0)=\left[\begin{array}{lllll}
-1 & 0.5 & 1 & 0.5 & -2
\end{array}\right]^{T} .
$$

The gain $Z$ is obtaind for $\gamma=22$ and $\mu_{1}=7.4628, \mu_{2}=$ 0.0057 and $\mu_{3}=0.2476$ and is then given by

$$
Z=\left[\begin{array}{llll}
-6179.565 & -6191.2468 & 47.380 & 47.2190 \\
-1810.724 & -1819.847 & 20.760 & 20.796
\end{array}\right] .
$$

Finally, the matrices of the reduced-order filter (5) are

$$
\begin{aligned}
M_{0} & =\left[\begin{array}{ll}
-9.291 & -4.791 \\
-4.862 & -7.362
\end{array}\right], & M_{1} & =\left[\begin{array}{cc}
-0.041 & -0.020 \\
-0.114 & -0.146
\end{array}\right], \\
N_{0} & =\left[\begin{array}{ll}
4.291 & -7.391 \\
5.862 & -3.262
\end{array}\right], & N_{1} & =\left[\begin{array}{cc}
0.171 & 0.009 \\
-0.002 & -0.002
\end{array}\right] .
\end{aligned}
$$

The following figures show the simulation results of the augmented system (32). The state $x(t)$ and the estimation error $e(t)$ are plotted. The disturbance signal $v(t)$ is presented with the error plots. The simulation is made for the control $u_{1}(t)=0.5 \sin (3 t)+2$, and the covariance factor between the Wiener processes defined in $(2 \mathrm{~b}) \varphi=0.0215$.

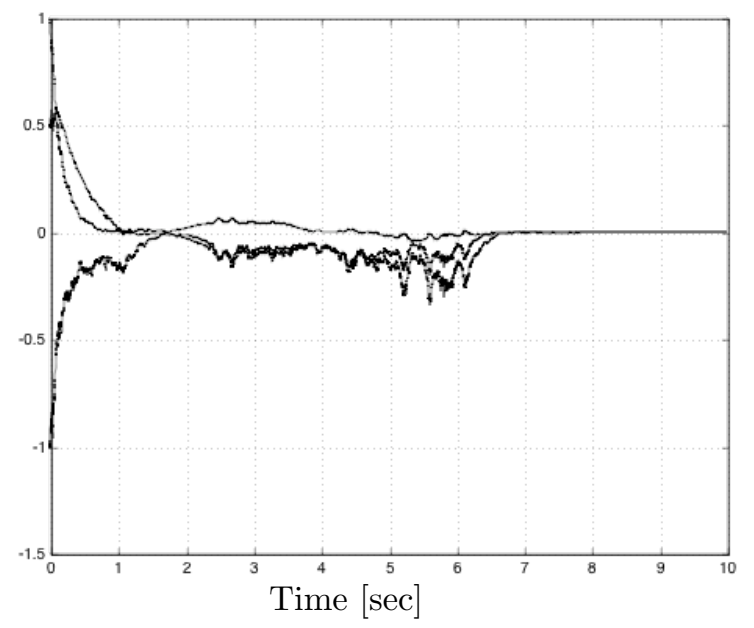

Fig. 1. The actual state $x(t)$.

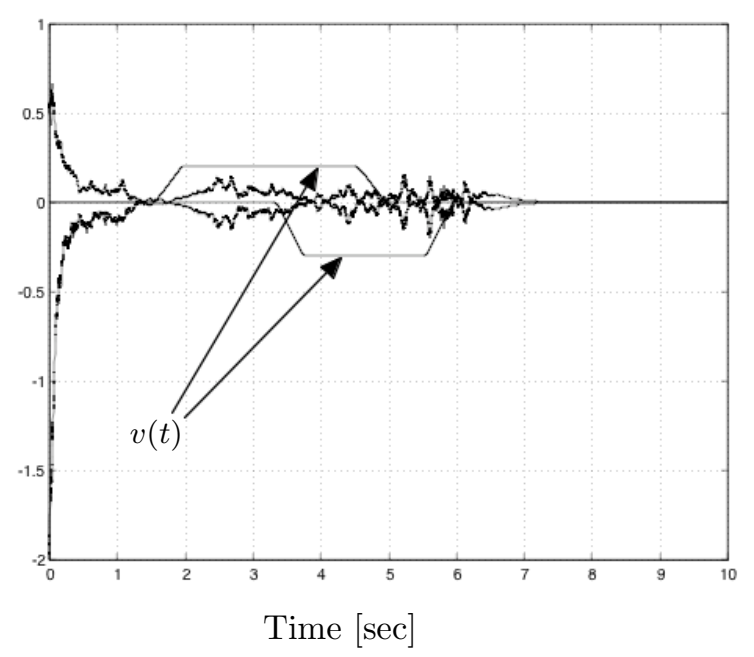

Fig. 2. The error $e(t)$ and the disturbance $v(t)$.

\section{Conclusion}

This paper provided a solution to the reduced-order $\mathcal{H}_{\infty}$ filtering problem for bilinear stochastic systems with multiplicative noise. The approach is based on a change of variable on the control input and on the using of a Sylvesterlike condition on the drift term to transform the problem into a robust reduced-order stochastic filtering one. Using the LMI method and the Itô formula we reduced the problem to the search of a unique gain matrix. Then the filter matrices are computed through this gain.

\section{REFERENCES}

Boyd, S.P., L. El Ghaoui, E. Féron and V. Balakrishnan (1994). Linear Matrix Inequality in Systems and Control Theory. SIAM. Philadelphia.

Carravetta, F., A. Germani and M.K. Shuakayev (2000). A new suboptimal approach to the filtering problem for bilinear stochastic differential systems. SIAM J. Contr. Opt. 38, 1171-1203.

Florchinger, P. (1995). Lyapunov-like techniques for stochastic stability. SIAM J. Contr. Opt. 33, 1151-1169.

Germani, A., C. Manes and P. Palumbo (2002). Linear filtering for bilinear stochastic differential systems with unknown inputs. IEEE Trans. Aut. Contr. 47, 1726-1730. 
Gershon, E., D.J.N. Limebeer, U. Shaked and I. Yaesh (2001). Robust $\mathcal{H}_{\infty}$ filtering of stationary continuous-time linear systems with stochastic uncertainties. IEEE Trans. Aut. Contr. 46, 17881793.

Has'minskii, R.Z. (1980). Stochastic Stability of Differential Equations. Siijthoff and Noordhoff. Aplhen aan den Rijn, The Netherlands.

Hinrichsen, D. and A.J. Pritchard (1998). Stochastic $\mathcal{H}_{\infty}$. SIAM J. Contr. Opt. 36, 1504-1538.

Kozin, F. (1969). A survey of stability of stochastic systems. Automatica 5, 95-112.

Lancaster, P. and M. Tismenetsky (1985). The Theory of Matrices. 2nd ed.. Academic Press. Orlando, USA.

Mao, X. (1997). Stochastic Differential Equations \& Applications. Horwood. London.

Souley Ali, H., H. Rafaralahy, M. Zasadzinski, S. Halabi and M. Darouach (2005). Observer design for a class of stochastic bilinear systems with multiplicative noise. In: Proc. IEEE American Contr. Conf.. Portland, USA.

Stoica, Adrian (2002). $\mathcal{H}_{\infty}$ filtering of signals subjected to multiplicative white noise. In: Proc. Triennal IFAC World Congress. Barcelona, Spain.

Wang, Y., L. Xie and C.E. De Souza (1992). Robust control of a class of uncertain nonlinear systems. Syst. \& Contr. Letters 19, 139149.

$\mathrm{Xu}, \mathrm{S}$. and T. Chen (2002). Reduced-order $\mathcal{H}_{\infty}$ filtering for stochastic systems. IEEE Trans. Sign. Proc. 50, 2998-3007.

$\mathrm{Xu}, \mathrm{S}$. and T. Chen (2003). Robust $\mathcal{H}_{\infty}$ filtering for uncertain impulsive stochastic systems under sampled measurements. Automatica 39, 500-516.

Zasadzinski, M., E. Magarotto, H. Rafaralahy and H. Souley Ali (2003). Residual generator design for singular bilinear systems subjected to unmeasurable disturbances : an LMI approach. Automatica 39, 703-713. 